\title{
Origin of resonant tunneling through single-point barriers
}

\author{
A.V. Zolotaryuk \\ Bogolyubov Institute for Theoretical Physics, National Academy of Sciences of Ukraine, \\ Kyiv 03143, Ukraine
}

\begin{abstract}
The physical interpretation of the appearance of resonant transmission through single-point barriers is discussed on the basis of a double-layer heterostructure in the squeezing limit as both the thickness of the layers and the distance between them tend to zero simultaneously. In this limit, the electron transmission through a barrier-well structure is derived to be non-zero at certain discrete values of the system parameters forming the so-called resonance set, while beyond this set, the structure behaves as a perfectly reflecting wall. The origin of this phenomenon is shown to result from the reflection coefficients at the interfaces in the inter-layer space. The transmission amplitude is computed as a set function defined on the trihedral angle surface in a three-dimensional parameter space.
\end{abstract}

Keywords:

One-dimensional quantum systems

Transmission

Point interactions

Resonant tunneling

\section{Introduction}

Since the pioneering studies [1, 2, 3] of resonant transmission through quantum multilayer heterostructures, electronic tunnel systems are a source of considerable interest. These structures are not only important in microand nanodevices, but their study involves a great deal of basic physics. In recent years it has been realized that the study of the electron transmission through heterostructures can be investigated in the zero-thickness limit

Preprint submitted to Physica E: Low-dimensional Systems and NanostructuresNovember 9, 2018 
approximation when their width shrinks to zero. Within such an approximation it is possible to produce the so-called point interaction models (see books [4, 5] for details and references) which are quite useful because they admit exact closed analytical solutions providing relatively simple situations, where an appropriate way of squeezing to zero can be chosen to be in relevance with a real structure.

The present paper focuses on the investigation of the physical mechanism of resonant tunneling through the planar heterostructure composed of extremely thin layers separated by some small distances in the limit as both the layer thickness and the distance between the layers simultaneously tend to zero. The electron motion in this system is confined in the longitudinal direction (say, along the $x$-axis), which is perpendicular to the planes, and is free in the transverse direction. The three-dimensional Schrödinger equation of such a structure can be separated into longitudinal and transverse parts, writing the total electron energy as the sum of the longitudinal and transverse energies: $E_{l}+\hbar^{2} \mathbf{k}_{t}^{2} / 2 m^{*}$, where $m^{*}$ is an effective electron mass and $\mathbf{k}_{t}$ the transverse wave vector, and expressing the wave function by the product $\psi=\psi_{l} \psi_{t}$. As a result, we arrive at the reduced one-dimensional Schrödinger equation with respect to the longitudinal component of the wave function $\psi_{l}(x)$ and the electron energy $E_{l}$. For brevity of notations, in the following we omit the subscript " $l$ " at both $\psi_{l}(x)$ and $E_{l}$. Thus, in the units as $\hbar^{2} / 2 m^{*}=1$, the one-dimensional stationary Schrödinger equation reads

$$
-\psi^{\prime \prime}(x)+V_{\varepsilon}(x) \psi(x)=E \psi(x),
$$

where the potential $V_{\varepsilon}(x)$ is given in terms of piecewise constant functions. It is supposed to depend on the squeezing parameter $\varepsilon>0$, so that in the limit as $\varepsilon \rightarrow 0$, the function $V_{\varepsilon}(x)$ is confined to one point.

A whole body of the mathematical and physical literature (see, e.g., [6, 7, 8, 9, 10, 11, 12, 13, 14, 15, 16, 17, 18, 19], a few to mention) has been published where a number of interesting features of point interactions was discovered for the one-dimensional Schrödinger equation with singular potentials in the form of distributions. In the work [20], it was suggested to regularize the potential $\gamma \delta^{\prime}(x), \delta^{\prime}(x):=d \delta(x) / d x$, where $\delta(x)$ is Dirac's delta function and $\gamma$ the potential strength (intensity), by a barrier-well potential profile $V_{\varepsilon}(x)$, and then perform the $\varepsilon \rightarrow 0$ limit. As a result, a non-zero transmission through this singular single-point barrier has been shown to occur under certain conditions imposed on the intensity $\gamma$ forming a discrete resonance 
set. Later on, it has been observed [21] that the resonance set for this point potential depends on the piecewise constant regularization of the distribution $\delta^{\prime}(x)$. Next, this family of point interactions has been extended using the $\delta^{\prime}$-like regularizing sequences of a more general type including those which are beyond piecewise constant functions [22]. Finally, it has rigorously been proved [23, 24, 25, 26, 27, 28] the existence of the resonance set for the potential $\gamma \delta^{\prime}(x)$ for arbitrary $\delta^{\prime}$-like regularizing sequence. Moreover, it has been derived that this set depends on the shape of the regularizing potential leading to the conclusion about the existence of a hidden parameter in the $\delta^{\prime}$-potential (see also [29]). Basically, e.g., in the works [29, 30, 31, 32], the realization of the one-point resonant-tunneling interactions has been treated as a cancellation of divergences in the squeezing limit. Therefore, it would be of interest to give the physical interpretation of the origin of this phenomenon and this is the main purpose of the present paper.

The procedure of looking for the resonance sets for the point interactions, which are realized from multilayer structures, can be described briefly as follows. Within each layer the potential $V_{\varepsilon}(x)$ is constant and therefore Eq. (11) can easily be solved. The solution can be represented via the transmission matrix connecting the boundary conditions for the wave function $\psi(x)$ at the left and right interfaces of the structure. Let the structure be located on the interval $\left(x_{1}, x_{2}\right)$. Then transmission matrix $\Lambda_{\varepsilon}$ is defined by the equations

$$
\left(\begin{array}{c}
\psi\left(x_{2}\right) \\
\psi^{\prime}\left(x_{2}\right)
\end{array}\right)=\Lambda_{\varepsilon}\left(\begin{array}{c}
\psi\left(x_{1}\right) \\
\psi^{\prime}\left(x_{1}\right)
\end{array}\right), \quad \Lambda_{\varepsilon}=\left(\begin{array}{cc}
\lambda_{11, \varepsilon} & \lambda_{12, \varepsilon} \\
\lambda_{21, \varepsilon} & \lambda_{22, \varepsilon}
\end{array}\right),
$$

where the matrix elements $\lambda_{i j, \varepsilon}$ satisfy the relation

$$
\lambda_{11, \varepsilon} \lambda_{22, \varepsilon}-\lambda_{12, \varepsilon} \lambda_{21, \varepsilon}=1 \text {, }
$$

being valid for any $\varepsilon>0$. As usual, the squeezing limit is arranged in such a way that $x_{1} \rightarrow-0$ and $x_{2} \rightarrow+0$ as $\varepsilon \rightarrow 0$.

In general, for any multilayer structure the limit relations $\lim _{\varepsilon \rightarrow 0} \lambda_{12, \varepsilon}=0$ and $\lim _{\varepsilon \rightarrow 0}\left|\lambda_{21, \varepsilon}\right|=\infty$ hold true. Under certain conditions on the system parameters, $\lim _{\varepsilon \rightarrow 0} \lambda_{11, \varepsilon}$ and $\lim _{\varepsilon \rightarrow 0} \lambda_{22, \varepsilon}$ can be finite and in this case the two-sided boundary conditions become of the Dirichlet type: $\psi( \pm 0)=0$. In physical terms, this means that the limit point structure acts as a perfectly reflecting wall. Since the element $\lambda_{21, \varepsilon}$ is the most singular term in the matrix $\Lambda_{\varepsilon}$, one can impose the constraint $\lim _{\varepsilon \rightarrow 0} \lambda_{21, \varepsilon}=0$ and, if this condition is satisfied, it can be viewed as an equation on the system parameters. At the 
parameter values satisfying this condition, the so-called resonance set, the transmission is non-zero (partial or perfect), while beyond this set the point structure is completely opaque.

In all the previous publications the condition $\lim _{\varepsilon \rightarrow 0} \lambda_{21, \varepsilon}=0$ was treated as a cancellation of divergences in the limit as $\varepsilon \rightarrow 0$, but nowhere the explanation of the origin of this phenomenon has been undertaken. This paper aims to explain how the resonant tunneling of this type happens in the simplest case of a double-layer structure. For this purpose we use the interference mechanism, similarly to that used in the works [33, 34], where instead of the description of point interactions in terms of the limiting transmission matrix $\Lambda:=\lim _{\varepsilon \rightarrow 0} \Lambda_{\varepsilon}$, an alternative way for identifying the whole family of point interactions has been used. This approach has been suggested in the works [11, 12], according to which the boundary conditions are written via the two-component vectors

$$
\Psi:=\left(\begin{array}{c}
\psi(+0) \\
\psi(-0)
\end{array}\right), \quad \Psi^{\prime}:=\left(\begin{array}{c}
\psi^{\prime}(+0) \\
-\psi^{\prime}(-0)
\end{array}\right) .
$$

The matrix equation for $\Psi$ and $\Psi^{\prime}$ reads

$$
(U-I) \Psi+\mathrm{i} L_{0}(U+I) \Psi^{\prime}=0,
$$

where $U \in \mathrm{U}(2)$ is a two-by-two unitary matrix, $I$ the unit matrix, and $L_{0}$ an arbitrary non-zero constant of length dimension. The $U$-matrix can be parametrized in an appropriate way and the relationship between its elements and the $\Lambda$-matrix elements can be established (for more details see [11]). Using this approach in [33, 34], the scattering of a quantum particle by two independent point interactions has been investigated in one dimension. As a result, the resonance conditions for perfect transmission through this twopoint system have been found.

The paper is organized as follows. In Section 2, we define the potential profile for a double-layer structure and derive the formulae for the reflectiontransmission coefficients. In the next section, the asymptotic representation of the resonance condition is obtained in the limit as the structure shrinks to one point. Based on the power-connecting three-scale parametrization of the system parameters, the transmission properties for a whole family of single-point interactions are investigated in Section 4. Finally, in Section 5, we give the concluding remarks. 


\section{Double-layer potential and reflection-transmission coefficients}

We consider the heterostructure composed of two homogeneous layers with width $l_{1}$ and $l_{2}$ separated at distance $r$. The potential for such a system can be expressed as the following piecewise constant function:

$$
V(x)=\left\{\begin{array}{l}
h_{1} \text { for } x_{1}<x<y_{1} \\
h_{2} \text { for } x_{2}<x<y_{2}, \\
0 \quad \text { for }-\infty<x<x_{1}, y_{1}<x<x_{2}, y_{2}<x<\infty
\end{array}\right.
$$

where $x_{1}<y_{1}<x_{2}<y_{2} ; l_{1}:=y_{1}-x_{1}, l_{2}:=y_{2}-x_{2}$ and $r:=x_{2}-y_{1}$. The transmission matrix $\Lambda_{j}$ for each layer $(j=1,2)$ is defined by the relations

$$
\left(\begin{array}{c}
\psi\left(y_{j}\right) \\
\psi^{\prime}\left(y_{j}\right)
\end{array}\right)=\Lambda_{j}\left(\begin{array}{c}
\psi\left(x_{j}\right) \\
\psi^{\prime}\left(x_{j}\right)
\end{array}\right), \quad \Lambda_{j}=\left(\begin{array}{ll}
\lambda_{j, 11} & \lambda_{j, 12} \\
\lambda_{j, 21} & \lambda_{j, 22}
\end{array}\right) .
$$

Each of these matrices connects the boundary conditions of the wave function $\psi(x)$ and its derivative $\psi^{\prime}(x)$ at $x=x_{j}$ and $x=y_{j}=x_{j}+l_{j}$. Explicitly,

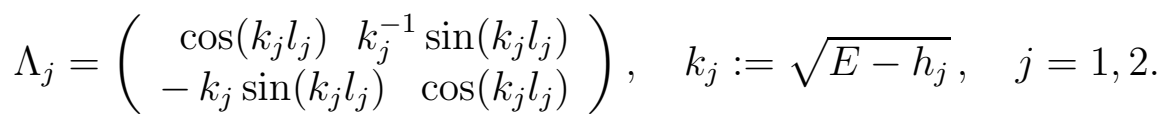

The scattering coefficients for each layer can directly be expressed through the elements of the $\Lambda$-matrices (8). The reflection and transmission coefficients for a quantum particle, incident from the left- and right-hand side and scattered by two layers (denoted by 1 and 2) can be defined by the following relations:

$$
\psi(x)= \begin{cases}\mathrm{e}^{\mathrm{i} k x}+R_{1}^{l} \mathrm{e}^{-\mathrm{i} k x} & \text { for }-\infty<x<x_{1}, \\ T_{1}^{l} \mathrm{e}^{\mathrm{i} k x} & \text { for } y_{1}<x<x_{2},\end{cases}
$$

for the plane wave $\exp (\mathrm{i} k x)$, incident from the left-hand side and scattered by layer 1 ,

$$
\psi(x)= \begin{cases}\mathrm{e}^{\mathrm{i} k x}+R_{2}^{l} \mathrm{e}^{-\mathrm{i} k x} & \text { for } \quad y_{1}<x<x_{2}, \\ T_{2}^{l} \mathrm{e}^{\mathrm{i} k x} & \text { for } y_{2}<x<\infty\end{cases}
$$

for the plane wave $\exp (\mathrm{i} k x)$, propagating in the inter-layer space and scattered by layer 2 , and

$$
\psi(x)= \begin{cases}\mathrm{e}^{-\mathrm{i} k x}+R_{2}^{r} \mathrm{e}^{\mathrm{i} k x} & \text { for } \quad y_{2}<x<\infty, \\ T_{2}^{r} \mathrm{e}^{-\mathrm{i} k x} & \text { for } y_{1}<x<x_{2},\end{cases}
$$


for the plane wave $\exp (-\mathrm{i} k x)$, incident from the right-hand side and scattered by layer 2 ,

$$
\psi(x)= \begin{cases}\mathrm{e}^{-\mathrm{i} k x}+R_{1}^{r} \mathrm{e}^{\mathrm{i} k x} & \text { for } \quad y_{1}<x<x_{2}, \\ T_{1}^{r} \mathrm{e}^{-\mathrm{i} k x} & \text { for }-\infty<x<x_{1},\end{cases}
$$

for the plane wave $\exp (-\mathrm{i} k x)$, propagating in the inter-layer space and scattered by layer 1 . Inserting the boundary conditions derived from the definition (9)-(12) into the matrix equations (7) with Eqs. (8), we find the following representation of the scattering coefficients in terms of the $\Lambda_{j}$-matrices:

$$
\begin{aligned}
R_{j}^{l} & =-\left(u_{j}+\mathrm{i} v_{j}\right) D_{j}^{-1} \mathrm{e}^{2 \mathrm{i} k x_{j}}, \quad R_{j}^{r}=\left(u_{j}-\mathrm{i} v_{j}\right) D_{j}^{-1} \mathrm{e}^{-2 \mathrm{i} k y_{j}}, \\
T_{j}^{l} & =T_{j}^{r}=2 D_{j}^{-1} \mathrm{e}^{\mathrm{i} k\left(x_{j}-y_{j}\right)},
\end{aligned}
$$

where

$$
\begin{gathered}
u_{j}=\lambda_{j, 11}-\lambda_{j, 22}=0, \quad v_{j}=k \lambda_{j, 12}+k^{-1} \lambda_{j, 21}=\left(\frac{k}{k_{j}}-\frac{k_{j}}{k}\right) \sin \left(k_{j} l_{j}\right), \\
D_{j}=\lambda_{j, 11}+\lambda_{j, 22}+\mathrm{i}\left(k^{-1} \lambda_{j, 21}-k \lambda_{j, 12}\right) \\
=2 \cos \left(k_{j} l_{j}\right)-\mathrm{i}\left(\frac{k}{k_{j}}+\frac{k_{j}}{k}\right) \sin \left(k_{j} l_{j}\right) .
\end{gathered}
$$

Consider now the plane wave $\exp (\mathrm{i} k x)$, incident upon the whole (doublelayer) structure from the left and thus the interference effect is present. Similarly, we define the scattering coefficients for the whole system located on the interval $\left(x_{1}, y_{2}\right)$ :

$$
\psi(x)= \begin{cases}\mathrm{e}^{\mathrm{i} k x}+R^{l} \mathrm{e}^{-\mathrm{i} k x} & \text { for }-\infty<x<x_{1}, \\ T^{l} \mathrm{e}^{\mathrm{i} k x} & \text { for } y_{2}<x<\infty .\end{cases}
$$

Then summing up all the trajectories according to Fig.1, we derive the following relations for the total reflection and transmission coefficients defined through Eq. (15):

$$
R^{l}=R_{1}^{l}+\frac{T_{1}^{l} T_{1}^{r} R_{2}^{l}}{1-R_{1}^{r} R_{2}^{l}} \quad \text { and } \quad T^{l}=\frac{T_{1}^{l} T_{2}^{l}}{1-R_{1}^{r} R_{2}^{l}}
$$

\section{Squeezing limit}

Using the explicit representation for the scattering coefficients, which follow from Eqs. (13) and (14), i.e.,

$$
R_{j}^{l}=\frac{\left(k / k_{j}-k_{j} / k\right) \exp \left(2 \mathrm{i} k x_{j}\right)}{k / k_{j}+k_{j} / k+2 \mathrm{i} \cot \left(k_{j} l_{j}\right)}, \quad R_{j}^{r}=\frac{\left(k / k_{j}-k_{j} / k\right) \exp \left(-2 \mathrm{i} k y_{j}\right)}{k / k_{j}+k_{j} / k+2 \mathrm{i} \cot \left(k_{j} l_{j}\right)},
$$




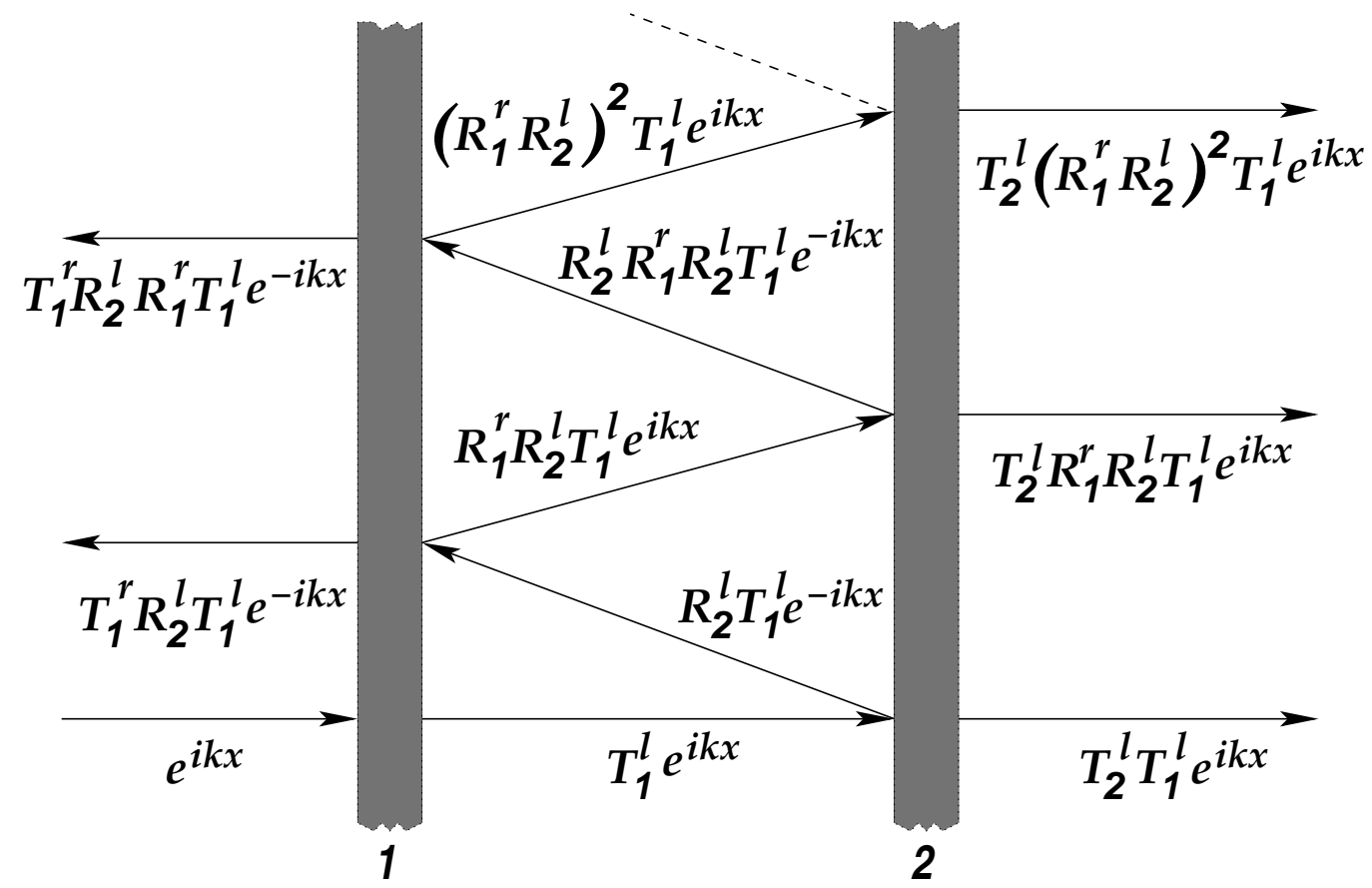

Figure 1: Schematic representation of the first several reflected and transmitted trajectories for the incident plane wave $\exp (\mathrm{i} k x)$ from the left-hand side, which are scattered by both layers 1 and 2. These trajectories correspond to the definition (9)-(12) and their summing up leads to the formulae (16). 


$$
T_{j}^{l}=T_{j}^{r}=\frac{\exp \left(-\mathrm{i} k l_{j}\right)}{\cos \left(k_{j} l_{j}\right)-(\mathrm{i} / 2)\left(k / k_{j}+k_{j} / k\right) \sin \left(k_{j} l_{j}\right)},
$$

one can estimate separately in the squeezing limit the numerator $T_{1}^{l} T_{2}^{l}$ and the denominator $1-R_{1}^{r} R_{2}^{l}$ in the second formula (16) for the total transmission $T^{l}$. In this limit, we have $l_{j} \rightarrow 0$ and $\left|h_{j}\right| \rightarrow \infty$, but the products $k_{j} l_{j}$, $j=1,2$, as the arguments of the trigonometric functions, must be finite (also including the zero limit). Hence, $k_{j} l_{j} \rightarrow \sqrt{-h_{j}} l_{j}=: A_{j} ; A_{j}$ 's are either real or imaginary, finite or zero. Therefore $k_{j} \rightarrow A_{j} / l_{j}$ and thus $k / k_{j} \pm k_{j} / k \rightarrow \pm A_{j} / k l_{j}$. As a result, we obtain the following asymptotics:

$$
\begin{aligned}
T_{1}^{l} T_{2}^{l} \rightarrow & \frac{\exp \left[-\mathrm{i} k\left(l_{1}+l_{2}\right)\right]}{\cos A_{1} \cos A_{2}\left(1-\mathrm{i} / 2 k \alpha_{1}\right)\left(1-\mathrm{i} / 2 k \alpha_{2}\right)}=\mathcal{O}\left(l_{1} l_{2}\right) \\
R_{1}^{r} R_{2}^{l} \rightarrow & \frac{\exp (2 \mathrm{i} k r)}{\left(1+2 \mathrm{i} k \alpha_{1}\right)\left(1+2 \mathrm{i} k \alpha_{2}\right)} \\
= & \left\{\left(1-4 k^{2} \alpha_{1} \alpha_{2}\right) \cos (2 k r)+2 k\left(\alpha_{1}+\alpha_{2}+2 \mathrm{i} k \alpha_{1} \alpha_{2}\right) \sin (2 k r)\right. \\
& \left.+\mathrm{i}\left[2 k\left(\alpha_{1}+\alpha_{2}\right) \cos (2 k r)-\sin (2 k r)\right]\right\}^{-1},
\end{aligned}
$$

where $\alpha_{j}:=\left(l_{j} / A_{j}\right) \cot A_{j} \rightarrow 0$ as $l_{j} \rightarrow 0$. As can be seen from the expression (18), the successive transmission through the layers does not depend on the inter-layer distance $r$ and it completely vanishes in the limit as $l_{1}, l_{2} \rightarrow 0$. Therefore, in general, the whole structure behaves as a fully reflecting wall. This is because of the bigger singularity than that of the typical double-delta potential [35]. Such a singularity occurs due to the non-zero finiteness of the arguments $A_{j}$.

The only possibility for the total transmission $T^{l}$ to be non-zero can happen if the denominator $1-R_{1}^{r} R_{2}^{l}$ will be of the same order $\mathcal{O}\left(l_{1} l_{2}\right)$ as the numerator (even in the particular case $r=0$ ). In general, this is impossible because of the presence of the sum $\alpha_{1}+\alpha_{2}$ in (19), so that the denominator appears to be of the order $\mathcal{O}\left(l_{1}, l_{2}\right)$. However, here one can impose the condition

$$
\sin (2 k r)=2 k\left(\alpha_{1}+\alpha_{2}\right) \cos (2 k r)
$$

and then the expression in the square brackets of (19) vanishes. Using next this equation in (19) once more, we get

$$
R_{1}^{r} R_{2}^{l} \rightarrow\left[1+4 k^{2}\left(\alpha_{1}^{2}+\alpha_{1} \alpha_{2}+\alpha_{2}^{2}\right)+8 \mathrm{i} k^{3}\left(\alpha_{1}+\alpha_{2}\right) \alpha_{1} \alpha_{2}\right] \cos (k r) .
$$

Thus, if we additionally assume here that $\cos (2 k r) \rightarrow 1$ or $\sin (k r) \rightarrow 0$, we obtain that $R_{1}^{r} R_{2}^{l}=1+\mathcal{O}\left(l_{1}^{2}, l_{2}^{2}, l_{1} l_{2}\right)$. Then Eq. (20) can be rewritten in the 
simple form:

$$
\tan (k r)=k\left(\alpha_{1}+\alpha_{2}\right),
$$

which can be viewed as the resonance condition on the system parameters $h_{j}, l_{j}, r ; j=1,2$. The particular case $r=0$ in this equation is also appropriate. Therefore the family of all solutions to Eq. (22) generates a discrete resonance set, at which the transmission amplitude occurs in the form of extremely sharp peaks like those shown, e.g., in Fig. 1 of the work [36] as a result of the (non-uniform) pointwise convergence as $l_{1}, l_{2}, \tan (k r) \rightarrow 0$. In other words, under the resonance condition (22), due to the infinite summing of the inter-layer back-forth reflection steps $R_{1}^{r} R_{2}^{l}$, we arrive at the uncertain ratio $0 / 0$ in the second formula (19). This uncertainty should be treated carefully by computing the full expressions for $R^{l}$ and $T^{l}$ and in this way one can confirm the validity of the resonance equation (22).

Thus, inserting Eqs. (17) into (16), we get

$$
R^{l}=-(u+\mathrm{i} v) D^{-1} \exp \left(2 \mathrm{i} k x_{1}\right), \quad T^{l}=2 D^{-1} \exp \left[-\mathrm{i}\left(l_{1}+l_{2}+r\right)\right],
$$

where

$$
\begin{aligned}
& u=\left(\frac{k_{2}}{k_{1}}-\frac{k_{1}}{k_{2}}\right) \sin \left(k_{1} l_{1}\right) \sin \left(k_{2} l_{2}\right) \cos (k r)+\left[\left(\frac{k}{k_{1}}-\frac{k_{1}}{k}\right) \sin \left(k_{1} l_{1}\right) \cos \left(k_{2} l_{2}\right)\right. \\
& \left.-\left(\frac{k}{k_{2}}-\frac{k_{2}}{k}\right) \cos \left(k_{1} l_{1}\right) \sin \left(k_{2} l_{2}\right)\right] \sin (k r), \\
& v=\left[\left(\frac{k}{k_{1}}-\frac{k_{1}}{k}\right) \sin \left(k_{1} l_{1}\right) \cos \left(k_{2} l_{2}\right)+\left(\frac{k}{k_{2}}-\frac{k_{2}}{k}\right) \cos \left(k_{1} l_{1}\right) \sin \left(k_{2} l_{2}\right)\right] \cos (k r) \\
& +\left(\frac{k_{1} k_{2}}{k^{2}}-\frac{k^{2}}{k_{1} k_{2}}\right) \sin \left(k_{1} l_{1}\right) \sin \left(k_{2} l_{2}\right) \sin (k r), \\
& D=\left[2 \cos \left(k_{1} l_{1}\right) \cos \left(k_{2} l_{2}\right)-\left(\frac{k_{1}}{k_{2}}+\frac{k_{2}}{k_{1}}\right) \sin \left(k_{1} l_{1}\right) \sin \left(k_{2} l_{2}\right)\right] \cos (k r) \\
& -\left[\left(\frac{k}{k_{1}}+\frac{k_{1}}{k}\right) \sin \left(k_{1} l_{1}\right) \cos \left(k_{2} l_{2}\right)+\left(\frac{k}{k_{2}}+\frac{k_{2}}{k}\right) \cos \left(k_{1} l_{1}\right) \sin \left(k_{2} l_{2}\right)\right] \sin (k r) \\
& \text { - i }\left\{\left[\left(\frac{k}{k_{1}}+\frac{k_{1}}{k}\right) \sin \left(k_{1} l_{1}\right) \cos \left(k_{2} l_{2}\right)+\left(\frac{k}{k_{2}}+\frac{k_{2}}{k}\right) \cos \left(k_{1} l_{1}\right) \sin \left(k_{2} l_{2}\right)\right] \cos (k r)\right. \\
& \left.+\left[2 \cos \left(k_{1} l_{1}\right) \cos \left(k_{2} l_{2}\right)-\left(\frac{k^{2}}{k_{1} k_{2}}+\frac{k_{1} k_{2}}{k^{2}}\right) \sin \left(k_{1} l_{1}\right) \sin \left(k_{2} l_{2}\right)\right] \sin (k r)\right\} .
\end{aligned}
$$

By direct calculations one can prove that $|D|^{2}=4+u^{2}+v^{2}$ and, as a result, 
the reflection-transmission amplitudes become

$$
\mathcal{R}^{l}:=\left|R^{l}\right|^{2}=\frac{u^{2}+v^{2}}{4+u^{2}+v^{2}} \quad \text { and } \quad \mathcal{T}^{l}:=\left|T^{l}\right|^{2}=\frac{4}{4+u^{2}+v^{2}}
$$

fulfilling the conservation law $\mathcal{R}^{l}+\mathcal{T}^{l}=1$ for the electron flow. Next, in the limit as $l_{1}, l_{2} \rightarrow 0$, we get the following asymptotic representation:

$$
\begin{aligned}
u \rightarrow & {\left[\left(\frac{l_{1}^{2}}{A_{1}^{2}}-\frac{l_{2}^{2}}{A_{2}^{2}}\right) \cos (k r)+\left(\alpha_{1}-\alpha_{2}\right) \frac{\sin (k r)}{k}\right] \frac{\cos A_{1} \cos A_{2}}{\alpha_{1} \alpha_{2}}, } \\
v \rightarrow & -\left[\left(\alpha_{1}+\alpha_{2}\right) \cos (k r)-\frac{\sin (k r)}{k}\right] \frac{\cos A_{1} \cos A_{2}}{k \alpha_{1} \alpha_{2}} \\
D \rightarrow & {\left[\left(2 \alpha_{1} \alpha_{2}-\frac{l_{1}^{2}}{A_{1}^{2}}-\frac{l_{2}^{2}}{A_{2}^{2}}\right) \cos (k r)-\left(\alpha_{1}+\alpha_{2}\right) \frac{\sin (k r)}{k}\right.} \\
& \left.+\frac{\mathrm{i}}{k}\left(\frac{\sin (k r)}{k}-\left(\alpha_{1}+\alpha_{2}\right) \cos (k r)\right)\right] \frac{\cos A_{1} \cos A_{2}}{\alpha_{1} \alpha_{2}} .
\end{aligned}
$$

Under the resonance condition (22), these asymptotics are finite. If additionally we assume $r \rightarrow 0$, this condition becomes

$$
r=\frac{l_{1}}{A_{1}} \cot A_{1}+\frac{l_{2}}{A_{2}} \cot A_{2}, \quad 0 \leq r<\infty,
$$

and, as a result, the asymptotics (28)-(30) reduce to the following simple expressions:

$$
u \rightarrow \theta-\theta^{-1}, \quad v \rightarrow 0, \quad D \rightarrow \theta+\theta^{-1}, \quad \theta:=-\frac{A_{1} l_{2} \sin A_{1}}{A_{2} l_{1} \sin A_{2}}
$$

The zero inter-layer distance $(r=0)$ can be considered as a particular case of the resonance condition (31). Clearly, the limiting expressions (28) and (29) satisfy Eqs. (27), retaining the conservation law $\mathcal{R}^{l}+\mathcal{T}^{l}=1$.

One of the important conclusions which immediately follows from the resonance equation (31) is the impossibility to realize a single-point interaction from the double-barrier system. Indeed, for both the barriers we set $A_{j}=\mathrm{i} \bar{A}_{j}$, where $\bar{A}_{j}:=\lim _{h_{j} \rightarrow \infty, l_{j} \rightarrow 0}\left(\sqrt{h_{j}} l_{j}\right)>0, j=1,2$, so that Eq. (31) reduces to

$$
r=-\sum_{j=1,2}\left(l_{j} / \bar{A}_{j}\right) \operatorname{coth} A_{j}
$$


Since $r>0$, whereas the right-hand side of Eq. (33) is negative, the zero squeezing of the distance between the barriers is forbidden and this result agrees with the studies in [32]. On the other hand, if one of the layers is a well, say the first one, the resonance equation reads

$$
r=\left(l_{1} / A_{1}\right) \cot A_{1}-\left(l_{2} / \bar{A}_{2}\right) \operatorname{coth} \bar{A}_{2},
$$

where both the terms $A_{1}$ and $\bar{A}_{2}$ are positive, so that Eq. (34) can be satisfied. Indeed, while varying $A_{1}$, one can examine the existence of a countable number of solutions to this equation.

One of the effective ways to analyze a whole family of single-point interactions in the limit as the parameters $l_{1}, l_{2}$ and $r$ tend to zero simultaneously is their representation through a single squeezing parameter $\varepsilon \rightarrow 0$ using different power-connecting relations. Below we will investigate the squeezing limit using a three-scale approach. In this way we generalize the family of point interactions obtained previously in several papers [20, 21, 29, 30, 31, 32].

\section{Power-connecting three-scale parametrization}

The resonance condition (31) is given in the asymptotic form as the layer thickness parameters $l_{1}, l_{2}$ and the inter-layer distance $r$ simultaneously shrink to one point. To proceed with the further analysis of the condition (31), one can simplify the one-point limit procedure by connecting these parameters through a single squeezing parameter $\varepsilon \rightarrow 0$. The natural connection can be done by using different powers of $\varepsilon$. Within such an approach, the three-scale parametrization [31] that connects the layer parameters through the parameter $\varepsilon>0$, can be used. Thus, we set

$$
h_{1}=a_{1} \varepsilon^{-\mu}, \quad h_{2}=a_{2} \varepsilon^{-\nu}, \quad l_{1}=\varepsilon, \quad l_{2}=\eta \varepsilon^{1-\mu+\nu}, \quad r=c \varepsilon^{\tau}, \quad c \geq 0,
$$

where $a_{j} \in \mathbb{R}, j=1,2$, and $\mu, \nu, \tau, \eta$ are arbitrary positive parameters. We denote the potential (6) parametrized by these relations by $V_{\varepsilon}(x)$. Our task is to describe the possible single-point interactions, which can be realized from all the limits $V_{\varepsilon}(x) \rightarrow \gamma \delta^{\prime}(x)$ (in the sense of distributions on the $C_{0}^{\infty}$ test functions).

The first step is to find the whole set in the $\{\mu>0, \nu>0, \tau>0\}$-octant, where the potential $\gamma \delta^{\prime}(x)$ can be defined in the standard distributional sense. To this end, we need to estimate in the limit as $\varepsilon \rightarrow 0$ the integral

$$
\left\langle V_{\varepsilon} \mid \varphi\right\rangle=\left(a_{1} \varepsilon^{-\mu} \int_{0}^{l_{1}}+a_{2} \varepsilon^{-\nu} \int_{l_{1}+r}^{l_{1}+r+l_{2}}\right) \varphi(x) d x
$$


for any $\varphi(x) \in C_{0}^{\infty}(\mathbb{R})$. Using the parametrization (35) and expanding $\varphi(\varepsilon \xi)=\varphi(0)+\varepsilon \xi \varphi^{\prime}(0)+\left(\varepsilon^{2} \xi^{2} / 2\right) \varphi^{\prime \prime}(\varsigma \varepsilon \xi)$, where $\xi=x / \varepsilon$ and $\varsigma \in(0,1)$ depends for a given $\varphi$ on $\varepsilon \xi$, the integral (36) can be computed explicitly. Thus, under the condition $a_{1}+\eta a_{2}=0(0<\eta<\infty)$, we get

$$
\left\langle V_{\varepsilon} \mid \varphi\right\rangle=-a_{1}\left(\frac{1}{2} \varepsilon^{2-\mu}+\frac{\eta}{2} \varepsilon^{2(1-\mu)+\nu}+c \varepsilon^{1-\mu+\tau}\right) \varphi^{\prime}(0)+R_{2}
$$

where the last term can be estimated as follows

$$
\begin{aligned}
\left|R_{2}\right| \leq \max _{\xi \in \mathbb{R}} \varphi^{\prime \prime}(\xi)\left|a_{1}\right|\left(\frac{1}{3} \varepsilon^{3-\mu}+\frac{\eta^{2}}{6} \varepsilon^{3(1-\mu)+2 \nu}+\frac{\eta}{2} \varepsilon^{3-2 \mu+\nu}\right. \\
\left.+\frac{\eta c}{2} \varepsilon^{2(1-\mu)+\nu+\tau}+c \varepsilon^{2-\mu+\tau}+\frac{c^{2}}{2} \varepsilon^{1-\mu+2 \tau}\right) .
\end{aligned}
$$

Under the inequalities

$$
1<\mu \leq 2, \quad 2(\mu-1) \leq \nu<\infty, \quad \mu-1 \leq \tau<\infty,
$$

it is easy to be convinced that all the powers of $\varepsilon$ [for the terms in the brackets of (38)] are positive. Therefore $R_{2} \rightarrow 0$ as $\varepsilon \rightarrow 0$ and the limit $V_{\varepsilon}(x) \rightarrow \gamma \delta^{\prime}(x)$ (in the sense of distributions), where the constant $\gamma \in \mathbb{R}$ is the intensity of the $\delta^{\prime}$-potential, leads to the relations

$$
a_{1}=2 \gamma / \zeta_{Q} \quad \text { and } \quad a_{2}=-2 \gamma / \eta \zeta_{Q}
$$

with the set function

$$
\zeta_{Q}=\lim _{\varepsilon \rightarrow 0}\left(\varepsilon^{2-\mu}+\eta \varepsilon^{2(1-\mu)+\nu}+2 c \varepsilon^{1-\mu+\tau}\right)= \begin{cases}1+\eta+2 c & \text { at } P, \\ \eta+2 c & \text { on } K, \\ 1+2 c & \text { on } L, \\ 1+\eta & \text { on } N, \\ \eta & \text { on } X, \\ 1 & \text { on } Y, \\ 2 c & \text { on } Z .\end{cases}
$$

Here $Q=P, K, L, N, X, Y, Z$ are the sets in the $(\mu, \nu, \tau)$-octant defined by

$\operatorname{vertex} P:=\{\mu=\nu=2, \tau=1\}$,

edge $K:=\{1<\mu<2, \nu=2(\mu-1), \tau=\mu-1\}$,

edge $L:=\{\mu=2,2<\nu<\infty, \tau=1\}$,

edge $N:=\{\mu=\nu=2,1<\tau<\infty\}$,

plane $X:=\{1<\mu<2, \nu=2(\mu-1), \mu-1<\tau<\infty\}$,

plane $Y:=\{\mu=2,2<\nu<\infty, 1<\tau<\infty\}$,

plane $Z:=\{1<\mu<2,2(\mu-1)<\nu<\infty, \tau=\mu-1\}$, 


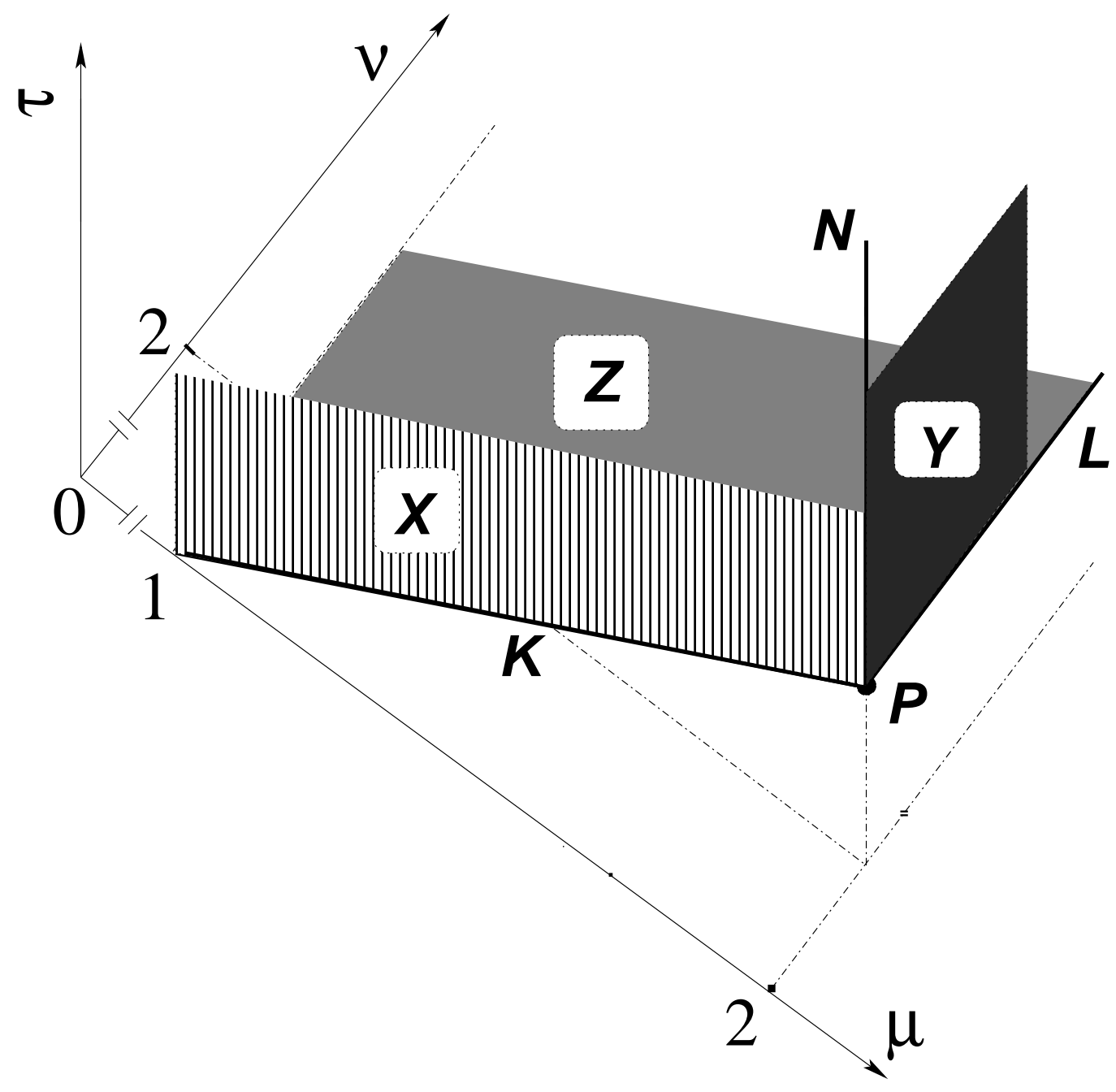

Figure 2: Trihedral angle surface $S_{\delta^{\prime}}=P \cup K \cup L \cup N \cup X \cup Y \cup Z$ formed by vertex $P$, three edges $K, L, N$ and three planes $X, Y, Z$, which are defined by Eqs. (42). 
and forming the trihedral angle surface $S_{\delta^{\prime}}$ as shown in Fig.2, Note that the inequalities (39) are fulfilled on this surface. According to Eqs. (40), on the plane $Z$, the distribution $\gamma \delta^{\prime}(x)$ makes sense only if $c>0$.

In general, on the whole $S_{\delta^{\prime}}$-surface, the resonance condition (31) parametrized by Eqs. (35) with the amplitudes (40) becomes an equation with respect to the intensity $\gamma$. Asymptotically, in the limit as $\varepsilon \rightarrow 0$, it takes the form

$$
\begin{gathered}
\sqrt{\eta} \varepsilon^{\nu / 2} \cot \left(\sqrt{2 \gamma \eta / \zeta_{Q}} \varepsilon^{1-\mu+\nu / 2}\right)-\varepsilon^{\mu / 2} \operatorname{coth}\left(\sqrt{2 \gamma / \zeta_{Q}} \varepsilon^{1-\mu / 2}\right) \\
=c \varepsilon^{\tau} \sqrt{2 \gamma / \zeta_{Q}} .
\end{gathered}
$$

Particularly, at the vertex $P$, this equation reduces to

$$
\sqrt{\eta} \cot \sqrt{\frac{2 \eta \gamma}{1+\eta+2 c}}=\operatorname{coth} \sqrt{\frac{2 \gamma}{1+\eta+2 c}}+c \sqrt{\frac{2 \gamma}{1+\eta+2 c}}
$$

and for the case $c=0$ we have the equation, which was derived in [21], i.e.,

$$
\tan \sqrt{\frac{2 \gamma \eta}{1+\eta}}=\sqrt{\gamma} \tanh \sqrt{\frac{2 \gamma}{1+\eta}} .
$$

If, additionally, $\eta=1$, we arrive at the most simple equation $\tan \sqrt{\gamma}=$ $\tanh \sqrt{\gamma}$, which was originally obtained in [20]. Clearly, these versions admit a countable number of roots $\gamma_{P, n}, n \in \mathbb{Z}$, forming the resonance set $\Sigma_{P}(\eta, c):=$ $\cup_{n=-\infty}^{\infty} \gamma_{P, n}$.

For the edges $K$ and $L$, in the limit as $\varepsilon \rightarrow 0$ Eq. (43) reduces to the following two equations:

$$
\begin{gathered}
\sqrt{\eta} \cot \sqrt{\frac{2 \gamma \eta}{\eta+2 c}}=\sqrt{\frac{\eta+2 c}{2 \gamma}}+c \sqrt{\frac{2 \gamma}{\eta+2 c}}, \\
\operatorname{coth} \sqrt{\frac{2 \gamma}{1+2 c}}=\sqrt{\frac{1+2 c}{2 \gamma}}-c \sqrt{\frac{2 \gamma}{1+2 c}},
\end{gathered}
$$

respectively. Equation (46) admits a countable number of solutions on the positive half-axis: $0 \leq \gamma_{K, n}<\infty$, while Eq. (47) on the negative half-axis $-\infty<\gamma_{L, n} \leq 0$. The resonance sets are $\Sigma_{K}(\eta, c):=\cup_{n=0}^{\infty} \gamma_{K, n}$ and $\Sigma_{L}(c):=$ $\cup_{n=0}^{\infty} \gamma_{L, n}$, respectively. Particularly, the resonance sets $\Sigma_{K}(\eta=1, c=0)$ and $\Sigma_{L}(c=0)$ are given by the roots of the simple equations

$$
\tan \sqrt{2 \gamma}=\sqrt{2 \gamma} \text { and } \tanh \sqrt{2 \gamma}=\sqrt{2 \gamma}
$$


respectively, found previously in [29]. On the $Z$-plane, Eq. (43) is fulfilled only for $c=0$, but in this case Eqs. (40) do not provide the $V_{\varepsilon}(x) \rightarrow \gamma \delta^{\prime}(x)$ limit.

On the edge $N$, Eq. (43) reduces to (45), so that $\Sigma_{P}(\eta, c=0)=\Sigma_{N}(\eta):=$ $\cup_{n=-\infty}^{\infty} \gamma_{N, n}$. Finally, on the planes $X$ and $Y$, the resonance sets $\Sigma_{X}$ and $\Sigma_{Y}$ are given by the roots of Eqs. (48), respectively, so that $\gamma_{X, n}=\gamma_{K, n}(\eta=1, c=$ $0)$ and $\gamma_{Y, n}=\gamma_{L, n}(c=0)$. Concerning the plane $Z$, where the distribution $\delta^{\prime}(x)$ is well defined, Eq. (43) for $Q=Z$ does not allow solutions except for $c=0$, but for this case the limit $V(x) \rightarrow \gamma \delta^{\prime}(x)$ cannot be defined.

Now the resonance condition (43) and its explicit representation given by Eqs. (44)-(48) can be used to compute the transmission amplitude $\mathcal{T}^{l}$ using Eqs. (27) and (32). Thus, the transmission amplitude as a set function of $Q$ and the $n$th resonance level can be rewritten in the form

$$
\mathcal{T}_{Q, n}^{l}=\frac{4 \theta_{Q, n}^{2}}{\left(1+\theta_{Q, n}^{2}\right)^{2}}
$$

with the asymptotics

$$
\begin{aligned}
& \theta_{Q, n}^{2} \rightarrow \eta \varepsilon^{\nu-\mu} \sinh ^{2}\left(\sqrt{2 \gamma_{Q, n} / \zeta_{Q}} \varepsilon^{1-\mu / 2}\right) / \sin ^{2}\left(\sqrt{2 \gamma_{Q, n} \eta / \zeta_{Q}} \varepsilon^{1-\mu+\nu / 2}\right) \\
&=\left(\cosh B_{Q, n}+c \varepsilon^{\tau-1} B_{Q, n} \sinh B_{Q, n}\right)^{2}+\eta \varepsilon^{\nu-\mu} \sinh ^{2} B_{Q, n} \\
& B_{Q, n}:=\sqrt{2 \gamma_{Q, n} / \zeta_{Q}} \varepsilon^{1-\mu / 2}, \quad \text { as } \varepsilon \rightarrow 0
\end{aligned}
$$

where Eq. (43) has been used. Explicitly, for each set $Q=P, K, L, N, X, Y$ and the $n$th resonance, we have

$$
\begin{aligned}
\theta_{P, n}^{2}= & \left(\cosh \sqrt{2 \gamma_{P, n} / \zeta_{P}}+c \sqrt{2 \gamma_{P, n} / \zeta_{P}} \sinh \sqrt{2 \gamma_{P, n} / \zeta_{P}}\right)^{2} \\
& +\eta \sinh ^{2} \sqrt{2 \gamma_{P, n} / \zeta_{P}}, \\
\theta_{K, n}^{2}= & \left(1+2 c \gamma_{K, n} / \zeta_{K}\right)^{2}+2 \eta \gamma_{K, n} / \zeta_{K}, \\
\theta_{L, n}^{2}= & \left(\cosh \sqrt{2 \gamma_{L, n} / \zeta_{L}}+c \sqrt{2 \gamma_{L, n} / \zeta_{L}} \sinh \sqrt{2 \gamma_{L, n} / \zeta_{L}}\right)^{2}, \\
\theta_{N, n}^{2}= & \cosh ^{2} \sqrt{2 \gamma_{N, n} / \zeta_{N}}+\eta \sinh ^{2} \sqrt{2 \gamma_{N, n} / \zeta_{N}}, \\
\theta_{X, n}^{2}= & 1+2 \gamma_{X, n}, \quad \theta_{Y, n}^{2}=\left(1-2 \gamma_{Y, n}\right)^{-1} .
\end{aligned}
$$

Particularly, using Eq. (49) and the first formula in (51), for $Q=P$ we obtain the following expression for the transmission amplitude at $c=0$ :

$$
\mathcal{T}_{P, n}^{l}(c=0)=\frac{\left(1-\tanh ^{2} \chi_{n}\right)\left(1+\eta \tanh ^{2} \chi_{n}\right)}{\left(1+\frac{\eta-1}{2} \tanh ^{2} \chi_{n}\right)^{2}}, \quad \chi_{n}:=\sqrt{\frac{2 \gamma_{P, n}(c=0)}{1+\eta}} .
$$


If additionally we set $\eta=1$, this equation reduces to the formula obtained in [20] with the resonance set $\left\{\gamma_{P, n}(\eta=1, c=0)\right\}_{n=-\infty}^{\infty}$ satisfying the resonance condition $\tan \sqrt{\gamma}=\tanh \sqrt{\gamma}, \gamma \in \mathbb{R}$.

\section{Concluding remarks}

Thus, the heterostructure consisting of two planar homogeneous layers has been investigated in the limit as their thickness parameters $l_{1}$ and $l_{2}$ tend to zero. As a result of this squeezing procedure, the asymptotic resonance condition (22) has been derived in a quite general form. Under this condition, the transmission through a double-layer structure has been observed to be non-zero at certain discrete values of the system parameters forming the so-called resonance set, while beyond this set, the structure behaves as a perfectly reflecting wall. Because of $l_{1}, l_{2} \rightarrow 0$, the limit $\tan (k r) \rightarrow 0$ must be accomplished as well. The particular case $\tan (k r)=0$ is also appropriate to satisfy the resonance condition. In other words, for the resonant tunneling to occur, the inter-layer distance must shrink sufficiently fast compared with the squeezing of the layer thickness.

In the case when $k r$ is found in the neighborhood of any point $n \pi$, $n=1,2, \ldots$, we deal with a two-point system. Then the condition (22) can be satisfied even for a typical double-barrier system if $k r<n \pi$ in this neighborhood. In this paper, we restrict ourselves to the limit case $r \rightarrow 0$, for which the resonance condition is asymptotically given by Eq. (31). The origin of the resonant tunneling in a squeezed double-layer heterostructure results from the requirement that each reflection step $R_{1}^{r} R_{2}^{l}$ at the interfaces in the inter-layer space must be of the order $1+\mathcal{O}\left(l_{1}^{2}, l_{2}^{2}, l_{1} l_{2}\right)$. This requirement provides in the squeezing limit the resonant-tunneling penetration through a barrier-well or a double-well system, but not for a double-barrier one.

Finally, using the three-scale parametrization (35), the transmission amplitude has been calculated as a set function defined on the trihedral angle surface shown in Fig. 2, where the potential $\gamma \delta^{\prime}(x)$ is defined in the sense of distributions. These calculations generalize the results derived in the previous publications.

\section{Acknowledgments}

The author acknowledges the partial financial support from the National Academy of Sciences of Ukraine (Project No. 0117U000238). He thanks Yaroslav Zolotaryuk for valuable suggestions and critical reading the manuscript. 


\section{References}

\section{References}

[1] R. Tsu, L. Esaki, Appl. Phys. Lett. 22 (1973) 562-564.

[2] L. Esaki, L.L. Chang, Phys. Rev. Lett. 33 (1974) 495-498.

[3] L.L. Chang, L. Esaki, R. Tsu, Appl. Phys. Lett. 24 (1974) 593-595.

[4] Y.N. Demkov, V.N. Ostrovskii, Zero-Range Potentials and Their Applications in Atomic Physics, Plenum Press, New York, 1988 (Leningrad University Press, Leningrad, 1975).

[5] S. Albeverio, F. Gesztesy, R. Høegh-Krohn, H. Holden, Solvable Models in Quantum Mechanics, second ed. with appendix by P. Exner, AMS Chelsea, RI, 2005.

[6] P. Šeba, Rep. Math. Phys. 24 (1986) 111-120.

[7] P. Kurasov, J. Math. Anal. Appl. 201 (1996) 297-323.

[8] S. Albeverio, L. Dąbrowski, P. Kurasov, Lett. Math. Phys. 45 (1998) 33-47.

[9] F.A.B. Coutinho, Y. Nogami, J.F. Perez, J. Phys. A: Math. Gen. 30 (1997) 3937-3945.

[10] F.A.B. Coutinho, Y. Nogami, L. Tomio, J. Phys. A: Math. Gen. 32 (1999) 4931-4942.

[11] T. Cheon, T. Fülöp, I. Tsutsui, Ann. Phys. (NY) 294 (2001) 1-23.

[12] I. Tsutsui, T. Fülöp, T. Cheon, J. Math. Phys. 42 (2001) 5687-5697.

[13] M. Gadella, J. Negro, L.M. Nieto, Phys. Lett. A 373 (2009) 1310-1313.

[14] R.-J. Lange J. High Energy Phys. JHEP11 (2012) 1-32.

[15] J.F. Brasche, L.P. Nizhnik, Methods Funct. Anal. Topol. 19 (2013) 4-15 (arXiv:1112.2545v1 [math.FA]). 
[16] M. Gadella, M.A. García-Ferrero, S. González-Martín, F.H. MaldonadoVillamizar, Int. J. Theor. Phys. 53 (2014) 1614-1627.

[17] R.-J. Lange, J. Math. Phys. 56 (2015) 122105 (17pp).

[18] M. Gadella, J. Mateos-Guilarte, J.M. Muñoz-Castañeda, L.M. Nieto, J. Phys. A: Math. Theor. 49 (2016) 015204 (22pp).

[19] S. Fassari, M. Gadella, M.L. Glasser, L.M. Nieto, Ann. Phys. (NY) 389 (2018) 4862.

[20] P.L. Christiansen, N.C. Arnbak, A.V. Zolotaryuk, V.N. Ermakov, Y.B. Gaididei, J. Phys. A: Math. Gen. 36 (2003) 7589-7600.

[21] A.V. Zolotaryuk, P.L. Christiansen, S.V. Iermakova, J. Phys. A: Math. Gen. 39 (2006) 9329-9338.

[22] F.M. Toyama, Y. Nogami, J. Phys. A: Math. Theor. 40 (2007) F685F690.

[23] Y.D. Golovaty, S.S. Man'ko, Ukrainian Math. Bull. 6 (2009) 169-203 (arXiv:0909.1034v1 [math.SP]).

[24] Y.D. Golovaty, R.O. Hryniv, J. Phys. A: Math. Theor. 43 (2010) 155204 (14pp) (A Corrigendum: J. Phys. A: Math. Theor. 44 (2011) 049802).

[25] Y. Golovaty, Methods Funct. Anal. Topol. 18 (2012) 243-255.

[26] Y.D. Golovaty, R.O. Hryniv, 2013 Proc. R. Soc. Edinb. A 143 (2013) 791-816.

[27] Y. Golovaty, Integr. Equ. Oper. Theor. 75 (2013) 341-362.

[28] Y. Golovaty, arXiv: 1801.09469v1 [math.SP] 29Jan2018.

[29] A.V. Zolotaryuk, Y. Zolotaryuk, Int. J. Mod. Phys. B 28 (2014) 1350203 (28pp).

[30] A.V. Zolotaryuk, Phys. Lett. A 374 (2010) 1636-1641.

[31] A.V. Zolotaryuk, Y. Zolotaryuk, J. Phys. A: Math. Theor. 44 (2011) 375305 (17pp) (A Corrigendum: J. Phys. A: Math. Theor. 45 (2012) $119501(2 \mathrm{pp}))$. 
[32] A.V. Zolotaryuk, J. Phys. A: Math. Theor. 50 (2017) 225303 (19pp).

[33] K. Konno, T. Nagasawa, R. Takahashi, Ann. Phys. (NY) 375 (2016) 91-104.

[34] K. Konno, T. Nagasawa, R. Takahashi, Ann. Phys. (NY) 385 (2017) 729-743.

[35] A. Laissaoui, L. Chetouani, J. Phys. A: Math. Theor. 59 (2018) 022106 (14pp).

[36] A.V. Zolotaryuk, Y. Zolotaryuk, J. Phys. A: Math. Theor. 48 (2015) 035302 (14pp). 